\title{
LncRNA CEBPA-AS1 Alleviates Cerebral Ischemia- Reperfusion Injury by Sponging miR-340-5p to Regulate APPL1/LKB1/AMPK Pathway
}

Xiankun Tu ( $\nabla$ unionnstu@hotmail.com )

Fujian Medical University Union Hospital

Huabin Zhang

Fujian Medical University Union Hospital

Bin Huang

Fujian Medical University Union Hospital

Xiyao Wu

Fujian Medical University Union Hospital

\section{Songsheng Shi}

Fujian Medical University Union Hospital

\section{Research}

Keywords: cerebral ischemia-reperfusion injury, CEBPA-AS1, miR-340-5p, APPL1/LKB1/AMPK pathway, MCAO/IR rat model

Posted Date: June 9th, 2020

DOl: https://doi.org/10.21203/rs.3.rs-32933/v1

License: (c) (i) This work is licensed under a Creative Commons Attribution 4.0 International License. Read Full License 


\section{Abstract}

BACKGROUND: The aim of this study was to research the mechanism of IncRNA CEBPA-AS1 in cerebral ischemia-reperfusion injury (CIRI).

METHODS: Middle cerebral artery occlusion (MCAO), MCAO/IR and OGD/R models were constructed. RNA immunoprecipitation (RIP) detected the binding of miR-340-5p to CEBPA-AS1. CEBPA-AS1 and miR340-5p was knockdown or up-regulated. RT-PCR and western blot was processed to detect the expression of related genes and proteins. Brain tissue water content in each group was determined. Nissl staining in hippocampus of the brain and NeuN staining (green) assay was used to observe nerve damage and detect nerve cell survival, respectively. Cell viability was detected by MTT assay. Nerve cell survival was observed by immunofluorescence cytochemistry assay. LDH and MDA content were detected by kit. TUNEL was used to detect apoptosis. Luciferase reporter system was processed for verifying the binding sites. After overexpressing CEBPA-AS1 in PC-12 cells, RNA pulldown assay was performed. TTC assay was used to observe the general morphology of the brain.

RESULTS: Overexpression of CEBPA-AS1 attenuated MCAO/IR-induced nerve damage. Increased CEBPAAS1 expression reduced neuronal apoptosis in MCAO/IR model. Knockdown of CEBPA-AS1 aggravated cell damage OGD/R cell model. CEBPA-AS1 increased APPL1 expression via negatively regulating miR340-5p, and affected APPL1/LKB1/AMPK pathway. CEBPA-AS1 attenuated OGD/R-induced cell damage by reducing miR-340-5p levels.

CONCLUSIONS: LnCRNA CEBPA-AS1 could alleviate cerebral ischemia-reperfusion injury by sponging miR-340-5p to regulate APPL1/LKB1/AMPK pathway.

\section{Introduction}

Cerebrovascular disease seriously endangered human health, which was with the characteristics of mortality, disability and unpredictability ${ }^{1}$. Among them, ischemic brain injury patients accounted for most of the cerebral vascular disease ${ }^{2}$. Timely recovery of blood flow and reperfusion after ischemia was important for restoring blood oxygen supply in the ischemic brain tissue and maintaining the normal morphology and function of damaged brain tissue ${ }^{3}$. However, a long time of ischemia and the reperfusion treatment will further aggravated the degree of brain tissue damage, which was cerebral ischemia-reperfusion injury (CIRI) ${ }^{4}$. CIRI was a complex pathological and physiological process, which included a variety of mechanisms, such as inflammatory reactions, calcium overload, excessive formation of free radicals, and toxic effects of excitatory amino acids ${ }^{5}$. Various links and factors worked together to promote the formation of brain infarction and the destruction of nerve function after CIRI. So far, drugs that improved cerebral ischemia-reperfusion injury in clinic mainly included anticoagulation, antiplatelet aggregation, neuroprotective agents and antioxidant drugs ${ }^{6}$. However, the pathophysiology of cerebral ischemia-reperfusion injury is a multi-link, multi-factor, multi-channel enzymatic cascade. 
Thereby, In-depth study of molecular mechanisms is important for the discovering of targets and the targeted drugs designing of this disease.

Long-chain noncoding RNAs (IncRNAs) were involved in many biological processes, including epigenetic modification, cell differentiation, and apoptosis ${ }^{7}$. In previous studies, IncRNAs were unique biomarkers that might be related to physiological or disease states ${ }^{8}$. Several IncRNAs have been identified as biomarkers for many human cancers. In recent years, IncRNA CEBPA-AS1 was confirmed to play an important role in various life activities such as dose compensation effect, epigenetic regulation, cell cycle regulation and cell differentiation regulation, which was a hot research topic in genetics. In human gastric cancer (GC) tissues, IncRNA CEBPA-AS1 was found to be enriched, which could be regarded as a prognostic or diagnostic marker for GC patients ${ }^{9}$. Besides, Guo et al. referred that IncRNA CEBPA-AS1 improved proliferation of oral squamous cell carcinoma cells and inhibited migration, invasion and apoptosis by participating CEBPA/Bcl2 pathway ${ }^{10}$. Interestingly, He et al. ${ }^{11}$ identified IncRNA profiling, constructed miRNA-IncRNA-mRNA interaction network and confirmed that IncRNA CEBPA-AS1 had a key regulatory effect in ischemic stroke. Thereby, IncRNA CEBPA-AS1 was important for diagnosis and treatment of cerebral ischemia-reperfusion injury. However, the specific mechanism was unclear.

The distribution of cerebral blood vessels in rats is very similar to that in humans ${ }^{12}$. The internal carotid artery and vertebral-basal artery form an arterial ring at the bottom of the brain, and then the branches supply blood. Therefore, rats are ideal animals for the preparation of CIRI. In order to research the mechanism of IncRNA CEBPA-AS1 in CIRI disease, MCAO/IR rats and OGD/R cell lines models were constructed in this study. Besides, the related target genes, miRNAs and pathways were also identified. The study might provide a new target for diagnosis and treatment of CIRI.

\section{Materials And Methods}

\section{Experiment animals and grouping}

Healthy adult SD rats weighed 250-280 g were purchased from the Shanghai Laboratory Animal Center, Chinese Academy of Sciences. For each animal experiment, 20 SD rats were used, which were divided into 4 groups $(n=5)$. All rats were housed in a terrarium with $22-26^{\circ} \mathrm{C}$, a $12 \mathrm{~h}$ light/dark cycle and ad libitum feeding and water. Rats were randomly divided into a model group and a sham operation group. The model group was further divided into MCAO/IR-12 $\mathrm{h}$ group, MCAO/IR-24 $\mathrm{h}$ group, and MCAO/IR-48 $\mathrm{h}$ (5 rats in each group). All experiments in this study were approved by All animal experiments were conducted according to the NIH Guide for the Care and Use of Laboratory Animals and approved by the Institutional Animal Care and Use Committee of Fujian Medical University.

\section{Construction of middle cerebral artery occlusion (MCAO)}

The establish methods was referred to previous reports ${ }^{13}$. All rats were anesthetized by $3 \%$ isoflurane (Shanghai Xinfan Biological Technology Co., Itd. Shanghai, China) in 70/30\% medical air/oxygen at room 
temperature $\left(25^{\circ} \mathrm{C}, 360 \mathrm{rag} / \mathrm{ks}\right)$. The median of neck was incised, the left external carotid artery (ECA) was ligated, the common carotid artery (CCA) and the internal carotid artery (ICA) were placed in the aneurysm clip. After breaking the ECA, a $0.25 \mathrm{~mm}$ (4 to 0 ) diameter nylon thread coated with $0.1 \%$ of Lpolylysine at the end was inserted at a depth of 18-20 mm from the common carotid bifurcation. The animals were anesthetized again 2 hours later and the nylon thread was carefully pulled out. The rats of sham group were processed with a nylon thread insertion by depth of $15 \mathrm{~mm}$ and the remaining steps are the same. The anus temperature of the rats was maintained at 36.5 to $37.5^{\circ} \mathrm{C}$ during the operation.

\section{Construction of OGD/R model}

In this experiment, the cell model of cerebral ischemia-reperfusion injury was simulated by means of glucose oxygen deprivation and re-glycemia and reoxygenation (OGD/R). PC12 cells were purchased from Shanghai Institute of Biosciences Cell Resource Center, Chinese Academy of Sciences. The specific manipulation steps are as follows. When the density reaches 80 to $90 \%$, cells was transferred to a cell culture dish and cultured overnight until the cells were attached to the wall. On the next day, the original medium was drained, fresh medium was replaced for control group. For the OGD experimental group, cells were washed with PBS (Mlbio Co., Itd. Shanghai, China) to wash the residual medium and cell debris, and a sufficient amount of sugar-free Eagle'S solution (Gibco Life Technologies, Inc., Grand Island, NY, USA) was added and transferred to an anoxic incubator (Shanghai Yuejin Medical Instruments CO., Ltd, Shanghai, China), in which PC12 cells was lacking glucose and hypoxia for $1 \mathrm{~h}$. Then the sugar-free Eagle's solution was discarded, and the complete medium was added. Cells were placed in a cell culture incubator with $5 \% \mathrm{CO}_{2}$ at $37^{\circ} \mathrm{C}$ to achieve complex sugar reoxygenation. The cells were collected after reoxygenation for 24 to 48 hours.

\section{Knockdown and overexpression of CEBPA-AS1}

The corresponding overexpression sequence and shRNAs (shRNA 1: 5'-GCGCGGATTCTCTTTCAAAGC-3', shRNA 2: 5'-GCACCGAGGGAGGAGACAAAC-3', shRNA 3: 5'-GCGTCCCTCGCATTCTTTACC-3') were designed according to the CEBPA-AS1 gene sequence of Genbank, and its sequence was synthesized by Wuhan Jingsai Bioengineering Technology Co., Ltd. For overexpression of CEBPA-AS1, the CEBPA-AS1 full-length IncRNA directed cloning into the multiple cloning site of the adenoviral vector. After correct identification by enzyme digestion, it was named Ad-CEBPA-AS1. The empty vector was used as control. Adenovirus overexpressing CEBPA-AS1 was injected into model rats. MiR-340-5p was simultaneously injected for rescue assay. For knockdown of CEBPA-AS1, shRNAs were transfected in OGD/R cells for 12, 24 and $48 \mathrm{~h}$. The processes of transfection were followed the operation manual of Lipofectamine ${ }^{\circledR} 2000$ (Invitrogen, Carlsbad, USA).

\section{QRT-PCR assay}

qRT-PCR was used to detect the expression of CEBPA-AS1 and miR-340-5p in brain tissue and cells after reperfusion. Total RNA were extracted from tissues or cells using the TRIzol reagent (Thermo Fisher Scientific, USA). $1 \mu \mathrm{g}$ total RNA was taken as a reverse transcription template. The following PCR primers were used: CEBPA-AS1 forward sequence, 5'-CGATGTGAGCCGGAGAG-3' and reverse, 5'- 
TTGATTTCCGTCCAGGTCT-3'; miR-340-5p forward sequence, 5'-AGGCGCTTATAAAGCAATGAGA-3' and reverse, 5'-GTGTGGTGTGGTATGGTGTG-3'. Reverse transcription was performed according to the instructions of K1622 reverse transcription kit. After reverse transcription, fluorescent quantitative PCR was performed on a QuantStudio 3 instrument using a QuantiNovaTM SYBR® Green PCR kit (QLAGEN, Germany), and the specific procedures of the experiment followed the corresponding kit instructions.

\section{TTC staining}

The left MCA of the rats was sacrificed for 2 hours after reperfusion for 24 hours, and the brain was decapitated. The general morphology of the brain was observed, the cerebellum and brainstem were removed and the sample was stayed in the physiological saline $\left(0\right.$ to $\left.4{ }^{\circ} \mathrm{C}\right)$ for 10 minutes. $2 \mathrm{Mm}$ thick coronal sections were placed in $2 \%$ TTC/phosphate buffer (Sigma, St Louis, MO), stayed in $37^{\circ} \mathrm{C}$ for 30 minutes in the dark, and fixed in $4 \%$ paraformaldehyde/PBS for 6 hours to observe the coloration of brain infarction by Olympus microscope (Olympus, Tokyo, Japan).

\section{Brain water content determination}

The rats were sacrificed by excessive anesthesia at the corresponding time points, and about $250 \mathrm{~g}$ of brain tissue was taken. After weighing the wet weight of each tissue with a JA1003 electronic balance, the sample was dried in an infrared drying oven at $100{ }^{\circ} \mathrm{C}$ for 24 hours, and the dry weight was weighed. The formula for calculating brain water content was as follows: brain water content = (wet weight - dry weight) / wet weight $\times 100 \%$

\section{Nissl staining assay}

The sample was routinely sliced and placed in a fume hood for $48 \mathrm{~h}$. Distilled water and glacial acetic acid were used to prepare a tar purple dye solution with a concentration of $0.001 \mathrm{~g} / \mathrm{ml}$, and the solution was centrifuged at $2000 \mathrm{r} / \mathrm{min}$ for $10 \mathrm{~min}$ to remove the precipitated particles for use. The slices were immersed in chloroform, absolute ethanol, $95 \%$ ethanol, $70 \%$ ethanol for $1 \mathrm{~min}$, soaked in distilled water for several seconds. The samples were then incubated in tar purple dye solution (Mlbio Co., Itd. Shanghai, China) at $37^{\circ} \mathrm{C}$ and room temperature for $15 \mathrm{~min}$, respectively. Then, the slices were washed with distilled water for $2 \mathrm{~min}$, and separated by $95 \%$ alcoholic acetic acid solution for 3 times. The sections were transparent by xylene for $5 \mathrm{~min}$, and sealed with neutral gum. After drying, the sample was observed under the light microscope (Jena, Germany) and photographed.

\section{Immunofluorescence cytochemistry assay}

The coverslips with cell growth for 7 days were rinsed 3 times with PBS. The cells were fixed in $40 \mathrm{~g} / \mathrm{L}$ paraformaldehyde for $30 \mathrm{~min}$, washed three times with PBS, $5 \mathrm{~min} /$ time, and blocked with $0.1 \%$ BSA for $1 \mathrm{~h}$. Rabbit anti-rat NeuN antibody (Upstate, Billerica, MA) was added, the sample was incubated for $1 \mathrm{~h}$ at room temperature and washed with PBS for 3 times. The secondary antibody plus rhodamine labeled goat anti-rabbit IgG antibody (Abcam, UK) with an antibody concentration of 1:200 was added. After the reaction was completed, the cell-grown surface of the cover slip was placed on a glass slide in which a 
mixture of glycerin and PBS (1:1) was dropped. Nerve cell survival was observed by a fluorescence microscope (Oly mpus, BX 60).

\section{Detection of lactate dehydrogenase (LDH)}

The LDH activity in serum was detected by LDH kit (Clontech, Mountain View, CA, USA) following the instructions. Serum diluted in saline at a volume ratio of 1:10. Total $10 \mu \mathrm{L}$ sample was added to the microplate. The substrate buffer and the oxidized coenzyme I solution were mixed at a volume ratio of $5: 1,60 \mu$ of the mixed solution was added to each well. The sample was thoroughly mixed and stayed in $37^{\circ} \mathrm{C}$ for 15 minutes. Add $50 \mu$ of 2,4-dinitrophenylhydrazine solution and mix well with the sample. 37 degrees Celsius water bath for 15 minutes. $150 \mu$ l of stop reagent were added to samples and mixed well. After standing at room temperature for 3 minutes, the wavelength was measured at $440 \mathrm{~nm}$.

\section{Detection of malondialdehyde (MDA) content}

The content of MDA was detected by MDA kit (Jiancheng, Nanjing, China) following the instructions. The brief steps were as follows. Cells were collected into a centrifuge tube, centrifuged, and the supernatant was discarded. The ratio of cells $\left(10^{4}\right)$ to the volume of the extract $(\mathrm{ml})$ is $500 \sim 1000: 1$. The cells were disrupted by sonication, centrifuged at $8000 \mathrm{~g}$ for 4 minutes at $4^{\circ} \mathrm{C}$, and the supernatant was taken and placed on ice for testing. $0.3 \mathrm{ml}$ of the reagent was placed in a centrifuge tube, and $0.1 \mathrm{ml}$ of the sample was added and mixed. The sample was placed in a water bath for $30 \mathrm{~min}$, cooled in an ice bath, and centrifuged at $25^{\circ} \mathrm{C}$ for 10 minutes. $200 \mu$ of the supernatant was pipetted into a 96 -well plate, and the absorbance at $532 \mathrm{~nm}$ and $600 \mathrm{~nm}$ was measured. The MDA content was calculated by the absorbance.

\section{FISH assay for distribution of IncRNA CEBPA-AS1 in cells}

The cell slide was placed on the bottom of a 24-well plate. A total of $5 \times 103$ cells were added into per well, and the supernatant was removed after $24 \mathrm{~h}$ of culture. Cells were washed with PBS. After being fixed with 4\% paraformaldehyde, PBS containing 0.5\% TritonX100 was added, and the pre-hybridization solution was used to block at $37^{\circ} \mathrm{C}$. The IncRNA CEBPA-AS1 probe was applied to hybridize overnight at $37^{\circ} \mathrm{C}$, and the hybridization washing solution was used to wash the cells at $42^{\circ} \mathrm{C}$. The hybridization area was stained with DAPI, and the slide was fixed in the dark. The samples were observed under a laser confocal microscope (OLYMPUS JAPAN CO., LTD, Japan).

\section{Detection of apoptosis in nerve cells by TUNEL assay}

Nerve cells were seeded in a laser confocal glass dish at $1 \times 10^{6}$ cells per well, and cultured in a humidity incubator for $24 \mathrm{~h}$. The sample was treated by $100 \mathrm{ul} 20 \mu \mathrm{g} / \mathrm{ml}$ Proteinase $\mathrm{K}$ for cell permeability. The sample was immersed in $4 \%$ paraformaldehyde for 5 minutes to fix. Total 100 ul of equilibration solution was added and placed in a wet box to equilibrate for $10 \mathrm{~min}$. $100 \mu \mathrm{l}$ of TUNEL reaction mixture was added to the specimen and reacted for $1 \mathrm{~h}$ at $37^{\circ} \mathrm{C}$. The reaction was stopped by immersing in SSC for 15 min. After blocking and dipping, DAB was processed in the dark. The samples were counterstained with hematoxylin, dehydrated with gradient alcohol, transparent twice with xylene, and sealed with neutral gum. Finally, the sample was observed and photographed. 


\section{Immunofluence assay}

After paraffin sections were dewaxed and gradient alcohol dehydrated, antigen retrieval was performed. The samples were then rinsed with 0.01M PTST for 5 minutes, placed in $2 \%$ BSA and seal in a wet box at $37^{\circ} \mathrm{C}$ for 30 minutes. Appropriately diluted fluorescently labeled antibodies were added dropwise to the samples and incubated for 30 minutes. Appropriately diluted fluorescently labeled antibodies were added dropwise to the samples and incubated for 30 minutes. Buffered glycerol is used for mounting slides. The samples were observed under a fluorescence microscope (Oly mpus, BX 60).

\section{MTT assay}

Transfected PC-12 cells were incubated with $5 \times 10^{3}$ per well in 96-well plates overnight. After culturing the cells for $48 \mathrm{~h}, 20 \mu \mathrm{L}$ MTT ( $5 \mathrm{mg} \cdot \mathrm{mL}^{-1}$, Sigma-Aldrich, St. Louis, MO) was added and cultured in a $37^{\circ} \mathrm{C}$ incubator for $4 \mathrm{~h}$. The medium in the well was removed, $100 \mu \mathrm{L}$ of dimethyl sulfoxide was added, and the $A$ value was measured at $490 \mathrm{~nm}$ after shaking and mixing. The cell viability results were expressed as the ratio of the A values of the transfected group to the control group.

\section{Western blot assay}

Western blot method was used to detect the expression of Bcl-2, Bax, cleaved-caspase-3, cleaved-PAPR, APPL1, N-LKB1, C-LKB1, p-AMPK and AMPK. Total cellular protein was extracted. Protein separation was performed by SDS-PAGE, electric transferred to PVDF membranes (Millipore, Billerica, MA, USA)., blocked by $5 \%$ skim milk powder for 1 hour, and washed by TBS. Primary antibody was added to the blocking solution at a ratio of 1:1000 and slowly shake at $4{ }^{\circ} \mathrm{C}$ overnight. The primary antibody was discarded, the membrane was washed by TBST, goat anti-rabbit IgG secondary antibody (Thermo Fisher Scientific) was added to membrane and stayed for $1 \mathrm{~h}$. The membrane was exposure by chemiluminescence. Quantity One software was applied to analyze the data of gray scale.

\section{Luciferase reporter system detects relative fluorescence values}

The binding site of CEBPA-AS1 and miR-340-5p were determined by the online prediction software miRDB. Targetscan predicted target genes binding to miR-340-5p. To demonstrating the binding site, a mutant sequence of the CEBPA-AS1 3'UTR deletion miR-340-5p binding site and a wild-sequence insertion reporter plasmid were designed. Co-transfection of miR-340 mimics and wild-type (CEBPA-AS1-wt / miR340-5p) or mutant (CEBPA-AS1-mut / miR-340-5p) recombination in 293T cells was processed using dual luciferase reporter assay system (Promega, Madison, WI, USA). qRT-PCR was used to detect transfection efficiency of miR-340-5p mimic. Luciferase reporter system detected relative fluorescence values. Following the same steps, the luciferase reporter assay was also used to confirm the target APPL1 of miR-340-5p.

\section{RNA pull-down assay}


CEBPA-AS1 was overexpressed in PC-12 cells, and RNA pulldown experiments were performed, followed by qRT-PCR to detect miR-340-5p enrichment. 5-bromo-UTP (BrU) labeled CEBPA-AS1, and pcDNA3.1 were synthesized. The labeled RNAs were combined with anti-BrU antibody. Extracted cytoplasmic was incubated with the RNA/antibody/beads mixture for $2 \mathrm{~h}$. Afterwards, the beads were washed by Buffer II. BrdU was used to elute RNA/protein complexes and mass spectrometry analysis was processed for detection. Proteins specific binding to LncRNA CEBPA-AS1 were ranked based on spectra counts.

\section{RNA immunoprecipitation (RIP) assay}

RNA immunoprecipitation (RIP, Millipore, Bedford, MA) detected the binding site of miR-340-5p and CEBPA-AS1. The RNA lysate was used to lyse the cells. $1.5 \mathrm{mg}$ of total protein per sample was taken, and $800 \mu \mathrm{l}$ of NT-2 buffer was added. $20 \mathrm{mg}$ of AG02 antibody or rabbit IgG was added at a ratio of 1:50. The washed sample was blotted dry, and then $10 \mu$ of $2 \times$ proteins was added, boiled at $95^{\circ} \mathrm{C}$ for $5 \mathrm{~min}$, placed on ice, and subjected to Western blotting with Input for immunoprecipitation. Trizol extracted Input-RNA samples were quantified, then $2 \mu \mathrm{g}$ of RIP-derived RNA was reverse-transcribed into cDNA, and real-time PCR was used to detect the expression of miR-340-5p and CEBPA-AS1 in the AG02 antibody complex.

\section{Statistical analysis}

Each experiment was repeated three times and data are presented as the mean \pm standard error (SD). Statistical analysis was processed by SPSS17.0 (SPSS, Inc., Chicago, IL, USA). One-way ANOVA was used to determine the difference among at least three groups. The different between two groups were calculated by a t-test. $\mathrm{P}<0.05$ was regarded as significant.

\section{Results}

\section{Increasing CEBPA-AS1 expression attenuated MCAO/IR- induced nerve damage}

$\mathrm{MCAO/IR}$ rat models were successfully constructed. Based on the model construction and transfection, the rats were divided into 4 groups: sham group, MCAO/IR group, MCAO/IR + Ad-Vector group and $\mathrm{MCAO} / \mathrm{RR}+\mathrm{Ad}-\mathrm{CEBPA}-\mathrm{AS} 1$ group. As shown in Fig. 1A, the expression of CEBPA-AS1 was significantly higher in MCAO/IR group than sham group. After Ad-CEBPA-AS1 transfection, the expression of CEBPAAS1 was increased. The infarct volume in MCAO/IR rat model was significantly larger than model. Interestingly, the infarct volume was decreased after CEBPA-AS1 was up-regulated (Fig. 1B). Besides, the infarct volume and neurological deficit score significantly higher in MCAO/IR models than control. Upregulated CEBPA-AS1 decreased both infarct volume and neurological deficit score (Fig. 1C and D). Simultaneously, surviving neurons was lower in MCAO/IR models, while up-regulated CEBPA-AS1 ameliorated MCAO/IR-induced nerve damage (Fig. 1F). Thereby, the expression of CEBPA-AS1 was closely related with MCAO/IR-induced nerve damage. 


\section{Increased CEBPA-AS1 expression reduced neuronal apoptosis in MCAO/IR model}

LDH and MDA kits were used to detect LDH and MDA levels, respectively. As shown in Fig. 2A and B, LDH activity in serum and the content of MDA was significantly higher in MCAO/IR models. After ad-CEBPAAS1 transfected, above indexes were decreased. TUNEL assay was processed to detect apoptosis of neuronal cells. The results of TUNEL assay was similarly with LDH and MDA levels. The percentage of tunel positive cells was more in MCAO/IR models, while up-regulated CEBPA-AS1 decreased the ratio (Fig. 2C). Immunohistochemical assay was used to detect the expression of cleaved-caspase-3 and cleaved-PARP. Both cleaved-caspase-3 and cleaved-PARP was up-regulated in MCAO/IR models, while the expression of two proteins were decreased after ad-CEBPA-AS1 transfection (Fig. 2D). Thence, we inferred that increased CEBPA-AS1 expression reduced neuronal apoptosis in MCAO/IR model.

\section{Knockdown of CEBPA-AS1 aggravated cell damage OGD/R cell model}

OGD/R cell model was successfully constructed. Based on the model and transfection, the cells were divided into control group, OGD/R group, OGD/R + Scramble group and OGD/R + shRNA3 group. After OGD/R cell model construction, the expression of CEBPA-AS1 was gradually higher as time goes on (Fig. 3A). FISH assay was used for detecting IncRNA CEBPA-AS1 distribution in cells. The results revealed that CEBPA-AS1 was distributed both in the nucleus and in the cytoplasm of OGD/R cells (Fig. 3B). ShRNAs (shRNA 1, shRNA 2, shRNA 3) were designed according to the CEBPA-AS1 gene sequence, and transfected into cells. As shown in Fig. 3C, the inhibition effect of shRNA3 was most significant. Thereby, shRNA3 was used for further experiments. MTT assay was processed for cell viability. The relative cell viability in OGD/R group was significantly lower. After knockdown of CEBPA-AS1, the relative cell viability was significantly decreased (Fig. 3D). Similarly with MCAO/IR model, the LDH activity in supernatant, MDA and tunel positive cells were higher in OGD/R cells, and down-regulated CEBPA-AS1 further improved the indexes of LDH, MDA and tunel positive cells (Fig. 3E, F and G). Moreover, the expression of Bax, cleaved-caspase3 and cleaved-PARP were significantly higher in OGD/R group, and highest in $\mathrm{OGD} / \mathrm{R}+$ shRNA3 group. However, the expression of Bcl-2 was with opposite trend (Fig. 3H). Based on above results, knockdown of CEBPA-AS1 might aggravate cell damage OGD/R cell model.

\section{CEBPA-AS1 negatively regulated miR-340-5p expression as a molecular sponge}

The binding sites of miR-340-5p and CEBPA-AS1 were predicted according to the miRDB website. The binding site was shown in Fig. 4A. Based on transfection, the 293T cells were grouped into CEBPA-AS1$\mathrm{wt}+$ miR mimics, CEBPA-AS1-wt + miR-340-5p, CEBPA-AS1-mut + miR mimics and CEBPA-AS1-mut + miR340-5p groups. The luciferase reporter system detected relative fluorescence values. The relative luciferase activity was lowest in CEBPA-AS1-wt + miR mimics group (Fig. 4B). The result of Fig. 4C 
showed that the transfection of CEBPA-AS1 was successful. The expression of miR-340-5p was enriched after overexpression of CEBPA-AS1 (Fig. 4D). RIP experiments were performed in PC-12 cells, and miR340-5p, CEBPA-AS1 levels in RISC complexes were detected by qRT-PCR. For the expression of miR-340$5 p$ and CEBPA-AS1, both relative RNA levels in Ant-Ago2 and Input were larger than Ant-IgG (Fig. 4E). The expression of miR-340-5p was inhibited and improved by CEPA-AS1 and shRNA3 transfection, respectively (Fig. 4F).

\section{APPL1 was the target of miR-340-5p}

Targetscan predicted target genes binding to miR-340-5p. The binding sites of APPL1 and miR-340-5p were shown in Fig. 5A. Dual luciferase reporter assay validated the target gene APPL1 of miR-340-5p. As the result, the relative luciferase activity was significantly lower in APPL1-wt + miR-340-5p mimics than other groups (Fig. 5B). After miR-340-5p inhibitor transfected, the expression level of miR-340-5p was decreased, which confirmed the transfection was successful (Fig. 5C). Up-regulated miR-340-5p decreased the expression of APPL1, while down-regulated miR-340-5p increased the expression of APPL1 (Fig. 5D). Thereby, miR-340-5p and APPL1 was with negative relationship. Moreover, up-regulated miR340-5p increased the expression of N-LKB1, while it decreased the expression of APPL1, C-LKB1 and pAMPK. MiR-340-5p inhibitor played opposite role. However, the expression of miR-340-5p did not affect the expression of AMPK (Fig. 5E).

\section{CEBPA-AS1 increased APPL1 expression via regulating miR-340-5p, and affected APPL1/LKB1/AMPK pathway}

After over-expression and knockdown of CEBPA-AS1 in PC-12 cells, WB detected the expression levels of APPL1, LKB1 (nucleus and intracellular), p-AMPK, AMPK. After transfected by shRNA3, the expression of APPL1, C-LKB1, p-AMPK was down-regulated, while the expression of N-LKB1 was up-regulated. Besides, CEBPA-AS1 transfection was with the opposite influence in the expression of above proteins. However, the expression of AMPK was not regulated by the level of CEBPA-AS1 (Fig. 6A). Interestingly, after transfected by CEBPA-AS1, the expression of APPL1, C-LKB1, p-AMPK was up-regulated, while the expression of N-LKB1 was down-regulated. When the expression of miR-340-5p was up-regulated, the effects of CEBPA-AS1 on the expression of above proteins were rescued (Fig. 6B). Thereby, CEBPA-AS1 increased APPL1 expression via regulating miR-340-5p, and affected APPL1/LKB1/AMPK pathway.

\section{CEBPA-AS1 attenuated OGD/R-induced cell damage by reducing miR-340-5p levels}

Rescue assay was processed in vivo and in vitro to verify that CEBPA-AS1 alleviated cerebral ischemiareperfusion injury by sponging miR-340-5p to regulate APPL1/LKB1/AMPK pathway. CEBPA-AS1 and miR-340-5p were simultaneous overexpressed in OGD/R-induced PC-12 cells and MCAO/IR models. Based on the transfection, the cells were divided into pc-DNA + miR mimics group, CEBPA-AS1 + miR 
mimic group and CEBPA-AS1 + miR-340-5p group. In CEBPA-AS1 + miR mimic group, the relative cell viability was significantly increased, while the LDH activity in supernatant, MDA content, and percentage of TUNEL positive cells were decreased. Interestingly, miR-340-5p could rescue the effects (Fig. 7A-D). Besides, after transfected by CEBPA-AS1, the expression of Bcl-2 was up-regulated, while the expression of Bax, cleaved-caspase-3 and cleaved-PARP were down-regulated. When the expression of miR-340-5p was up-regulated, the effects of CEBPA-AS1 on the expression of above proteins were rescued (Fig. 7E). Above all, CEBPA-AS1 could alleviate cerebral ischemia-reperfusion injury by sponging miR-340-5p to regulate APPL1/LKB1/AMPK pathway. In vivo experiments including TTC staining, brain water content, LDH, MDA and TUNELwere processed. As the results, up-regulated CEBPA-AS1 decreased infract volume, brain water content, LDH activity in serum, MDA level and tunel positive cells in MCAO/IR model, while miR-340-5p weakened the effects of CEBPA-AS1 (Fig. 7F-J)

\section{Discussion}

Cerebral ischemia-reperfusion injury was related with free radical production, intracellular calcium overloading, excitatory amino acid toxicity, high white blood cell aggregation and lacking of high-energy phosphate compounds ${ }^{14,15}$. For improving the cure rate of CIRI, more effectively target genes and related processes needed to be clarified. IncRNA CEBPA-AS1 was confirmed to be important for diagnosis and treatment of cerebral ischemia-reperfusion injury, but the molecular mechanism was unclear. In order to research the mechanism of IncRNA CEBPA-AS1 in CIRI disease, MCAO/IR rats and OGD/R cell lines models were successfully constructed in this study. As the results, CEBPA-AS1 was up-regulated in $\mathrm{MCAO} / \mathrm{IR}$ rats and OGD/R cell lines. Knockdown of CEBPA-AS1 aggravated OGD/R-induced cell damage. CEBPA-AS1 could negatively regulate the expression of miR-340-5p, target APPL1, regulate APPL1/LKB1/AMPK pathway, and alleviated OGD/R-induced cell damage. Up-regulated CEBPA-AS1 significantly aggravated neurological damage in the MCAO/IR rat model.

Various IncRNAs have been verified to affect the process of CIRI disease. For example, IncRNA H19 could activate autophagy, and further induce CIRI ${ }^{16}$. Besides, IncRNA C2dat1 was also confirmed to target CaMKIII, participate in NF-KB signaling pathway, and then promote neuronal survival ${ }^{17}$. In previous studies, IncRNA CEBPA-AS1 have been referred to be the diagnostic markers for different diseases, such as gastric cancer ${ }^{9}$, oral squamous cell carcinoma ${ }^{10}$ and ischemic stroke ${ }^{11}$. However, the mechanism of IncRNA CEBPA-AS1 in CIRI was unclear. In this study, CEBPA-AS1 was up-regulated in MCAO/IR rats and OGD/R cell lines. Thereby, it might be a novel target for diagnosis and treatment of CIRI.

Interestingly, IncRNA CEBPA-AS1 was confirmed to negatively regulate the expression of miR-340-5p and then target APPL1. MiR-340-5p played an important role in various diseases, such as thyroid cancer, breast cancer and melanoma ${ }^{18-20}$. In above studies, miR-340-5p was verified to regulate the expression of ABCB5, $\beta$-catenin and BMP4. Coincidentally, Xing et al. suggested that AKT signaling pathway regulated the expression of $\beta$-catenin, and further effected the progress of focal cerebral ischemia-reperfusion injury ${ }^{21}$. Besides, miR-340-5p negatively related with the expression of STAT3 ${ }^{22}$. 
By regulating the expression of STAT-1, cerebral ischemia-reperfusion injury could be significantly ameliorated ${ }^{23}$. Furthermore, miR-340-5p might play a role in the regulation of BMP4 expression by negative regulating the differentiation of rat NSCs into neuronal differentiation ${ }^{24}$. Commonly, the proliferation of endogenous neural stem cells was always lacking for self-repairing of central nervous system after brain ischemia ${ }^{25}$. Especially for young-adult animals, neural stem cells treatment could reduce ischemic brain injury, increase neurogenesis and angiogenesis in aged rat models ${ }^{26}$. Thereby, we inferred that MiR-340-5p was also important in CIRI disease. It might be related with repair of central nervous system after brain ischemia.

In this study, the online software targetscan predicted that APPL1 was the target of miR-340-5p. Moreover, vitro experiments also confirmed that miR-340-5p negatively regulated the expression of APPL1, and then affected APPL1/LKB1/AMPK pathways. It worth noting that APPL1 could interact with various signaling molecules, and the interaction ability make it to be a key factor in cell signaling ${ }^{27}$. In 2010, Xin et al. ${ }^{28}$ confirmed that APPL1 could regulate TAK1-MKK2-P38 MAPK pathway, and also participate in the response of adiponectin stimulation. Importantly, MAPK-ERK-p38 was an important signal transduction pathway between intracellular and external, which had potential effects on response to ischemia-reperfusion injury ${ }^{29}$. The good news was that adiponectin has been successfully used in ischemic stroke rat models by activating adipoR1-APPL1-LKB1-AMPK signaling pathway, and further reducing infarct area and atrophy of brain, inducing neurological function ${ }^{30}$. Based on the above information, APPL1 and APPL1/LKB1/AMPK pathways might be a novel target and potential mechanism for CIRI.

\section{Conclusion}

In conclusion, LncRNA CEBPA-AS1 could alleviate cerebral ischemia-reperfusion injury by sponging miR340-5p to regulate APPL1/LKB1/AMPK pathway. The study might provide a theoretical basis for diagnosis and treatment of CIRI.

\section{Declarations}

\section{Ethics approval and consent to participate}

The study is approved by the Institutional Animal Care and Use Committee of Fujian Medical University.

\section{Consent for publication}

Not applicable.

\section{Availability of data and material}

The data of this study is availability on a reasonable request. 


\section{Competing interests}

The authors declare that they have no competing interests.

\section{Funding}

The Joint Funds for the Innovation of Science and Technology of Fujian province of China (2018Y9004), the Natural Science Foundation of Fujian Province of China (2017J01204), and the Natural Science Foundation of Fujian Province of China (2018J01313) supported this work.

\section{Authors' contributions}

Xiankun Tu and Huabin Zhang contributed for the study design. Bin Huang, Xiyao Wu, and Songsheng Shi performed the experiments and analyzed the data. Xiankun Tu, Songsheng Shi and Huabin Zhang wrote the manuscript.

\section{Acknowledgements}

None

\section{References}

1. Wei $Y Y, F a n X N$, Wang S, Shi XM. [Status and progress of stimulating parameters in acupuncture treatment of ischemic cerebrovascular disease]. Acupuncture Research. 2008;33:287-9.

2. Jungsook C, Hyeong-Kyu L. Wogonin inhibits ischemic brain injury in a rat model of permanent middle cerebral artery occlusion. Biol Pharm Bull. 2004;27:1561-4.

3. Hossmann KA, Lechtape-Grüter $\mathrm{H}$, Hossmann V. The role of cerebral blood flow for the recovery of the brain after prolonged ischemia. Z Neurol.204,281 - 99(1973).

4. Zhang J, et al. The Possible Damaged Mechanism and the Preventive Effect of Monosialotetrahexosylganglioside in a Rat Model of Cerebral Ischemia-Reperfusion Injury. J Stroke Cerebrovasc Dis. 2015;24:1471-8.

5. Bie X, et al. Effects of gastrodin on amino acids after cerebral ischemia-reperfusion injury in rat striatum. Asia Pac J Clin Nutr. 2013;16:305-8.

6. Onwuekwe IO, Ezealaadikaibe B. Ischemic Stroke and Neuroprotection. Ann Med Health Sci Res.2,186 - 90(2013).

7. Rice PA. Roles of microRNAs and long-noncoding RNAs in human immunodeficiency virus replication. Wiley Interdiscip Rev RNA.6,661 - 70).

8. Kim J, Nan S, Ma XJ, Park E. Abstract. 1990: Single cell co-expression profiles of immunotherapy targets in the tumor microenvironment of NSCLC samples. Cancer Research.77,1990-(2017).

9. Ke D, et al. The combination of circulating long noncoding RNAs AK001058, INHBA-AS1, MIR4435$2 \mathrm{HG}$, and CEBPA-AS1 fragments in plasma serve as diagnostic markers for gastric cancer. 
Oncotarget.8,21516-25(2017).

10. Guo Y, et al. Long Non-coding RNA CEBPA-AS1 Correlates with Poor Prognosis and Promotes Tumorigenesis via CEBPA/Bcl2 in Oral Squamous Cell Carcinoma. Cancer Biol Ther. 2017;19:00-.

11. He W, Wei D, Chen S, Li S, Chen W. Altered long non-coding RNA transcriptomic profiles in ischemic stroke. Hum Gene Ther.29,hum.2017.064(2017).

12. Tomimoto H, Akiguchi I, Wakita H, Nakamura S, Kimura J. Distribution of NADPH-diaphorase in the cerebral blood vessels of rats: a histochemical study. Neuroscience Letters.156,105-8).

13. Chiang T, Messing RO, Chou WH. Mouse Model of Middle Cerebral Artery Occlusion. J Vis Exp.48, (2011).

14. Mei HS, Zhang YJ, Miao QF, Wang YL. Effect of dipfluzine on [Ca (2+)]_i level in rats with focal cerebral ischemia-reperfusion injury. Chinese Pharmaceutical Journal. 2005;40:1713-6.

15. Liu YR, et al. Effects of catalpol on ATPase and amino acids in gerbils with cerebral ischemia/reperfusion injury. Neurol Sci. 2014;35:1229-33.

16. Wang J, Cao B, Han D, Sun M, Feng J. Long Non-coding RNA H19 Induces Cerebral Ischemia Reperfusion Injury via Activation of Autophagy. Aging Disease. 2017;.8:71-84.

17. Xu Q, et al. Long non-coding RNA C2dat1 regulates CaMKIIठ expression to promote neuronal survival through the NF-KB signaling pathway following cerebral ischemia. Cell Death Dis. 2016;7:e2173.

18. Zhao P, et al. Up-regulation of miR-340-5p promotes progression of thyroid cancer by inhibiting BMP4. J Endocrinol Invest.1-8(2018).

19. Wozniak M, Sztiller-Sikorska M, Czyz M. Diminution of miR-340-5p levels is responsible for increased expression of ABCB5 in melanoma cells under oxygen-deprived conditions. Exp Mol Pathol.99,707 16(2015).

20. Du K, Li Z, Fang X, Cao T, Xu Y. Ferulic Acid Promotes Osteogenesis of Bone Marrow-Derived Mesenchymal Stem Cells by Inhibiting MicroRNA-340 to Induce $\beta$-catenin Expression through Hypoxia. Eur J Cell Biol.96,496(2017).

21. Xue-Song $X$, Fang $L$, Zhi-Yi H. Akt regulates $\beta$-catenin in a rat model of focal cerebral ischemiareperfusion injury. Mol Med Rep. 2015;11:3122-8.

22. Xiong Q, et al. Hepatitis B virus promotes cancer cell migration by downregulating miR-340-5p expression to induce STAT3 overexpression. Cell Biosci.7,16(2017).

23. Cai F, et al. Theaflavin ameliorates cerebral ischemia-reperfusion injury in rats through its antiinflammatory effect and modulation of STAT-1. Mediators Inflamm.2006,30490(2016).

24. Effect of miR. -340-5p targeting bone morphogenetic protein 4 on differentiation of rat neural stem cells. Chinese Journal of Behavioral Medicine Brain Science. 2017;26:395-9.

25. Zheng PD, et al. Ginkgolide B promotes the proliferation and differentiation of neural stem cells following cerebral ischemia/reperfusion injury, bothin vivoandin vitro. Neural Regen Res.13,1204(2018). 
26. Yaohui T, et al. Neural stem cell protects aged rat brain from ischemia-reperfusion injury through neurogenesis and angiogenesis. J Cerb Blood Flow Metab.34,1138(2014).

27. Li J, Mao X, Dong LQ, Liu F, Tong L. Crystal Structures of the BAR-PH and PTB Domains of Human APPL1. Structure.15,525 - 33(2007).

28. Xiaoban X, Lijun Z, Reyes CM, Feng L, Dong LQ. APPL1 mediates adiponectin-stimulated p38 MAPK activation by scaffolding the TAK1-MKK3-p38 MAPK pathway. Am J Physiol Endocrinol Metab. 2011;:300:E103.

29. Kovalska M, et al. Intracellular Signaling MAPK Pathway After Cerebral Ischemia-Reperfusion Injury. Neurochem Res. 2012;37:1568-77.

30. Xu N, et al. Adiponectin attenuates neuronal apoptosis induced by hypoxia-ischemia via the activation of AdipoR1/APPL1/LKB1/AMPK pathway in neonatal rats.

Neuropharmacology.133,S002839081830090 × (2018).

\section{Figures}



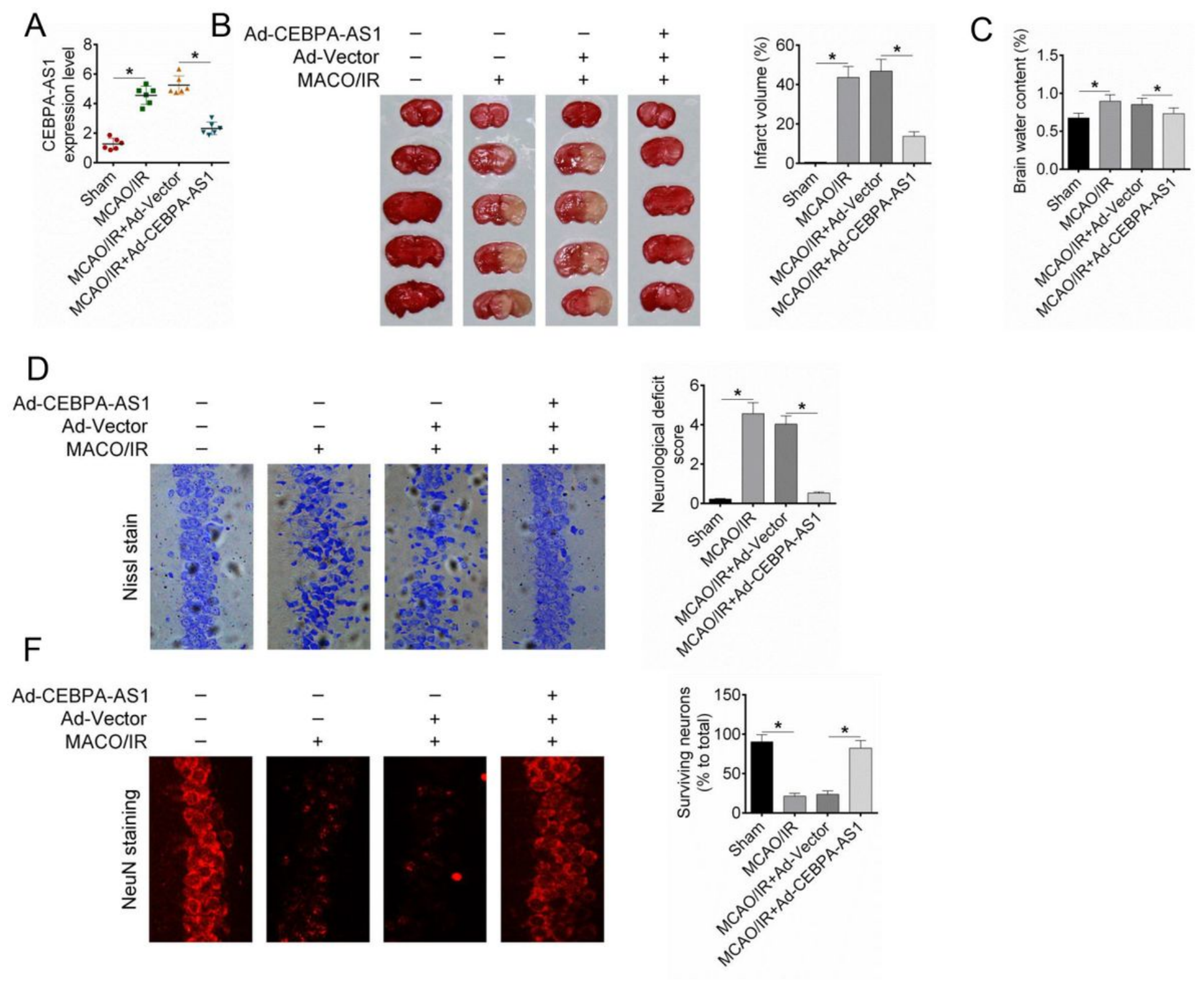

Figure 1

Increasing CEBPA-AS1 expression ameliorated MCAO/IR-induced nerve damage A. Detection of CEBPAAS1 expression level by RT-PCR; B. Brain region TTC staining and quantitative analysis of cerebral infarction volume; $\mathrm{C}$. Brain moisture content; D. Nissl staining in hippocampus of the brain to observe nerve damage; E. NeuN staining (green) detected nerve cell survival. ${ }^{*} \mathrm{P}<0.05$. 


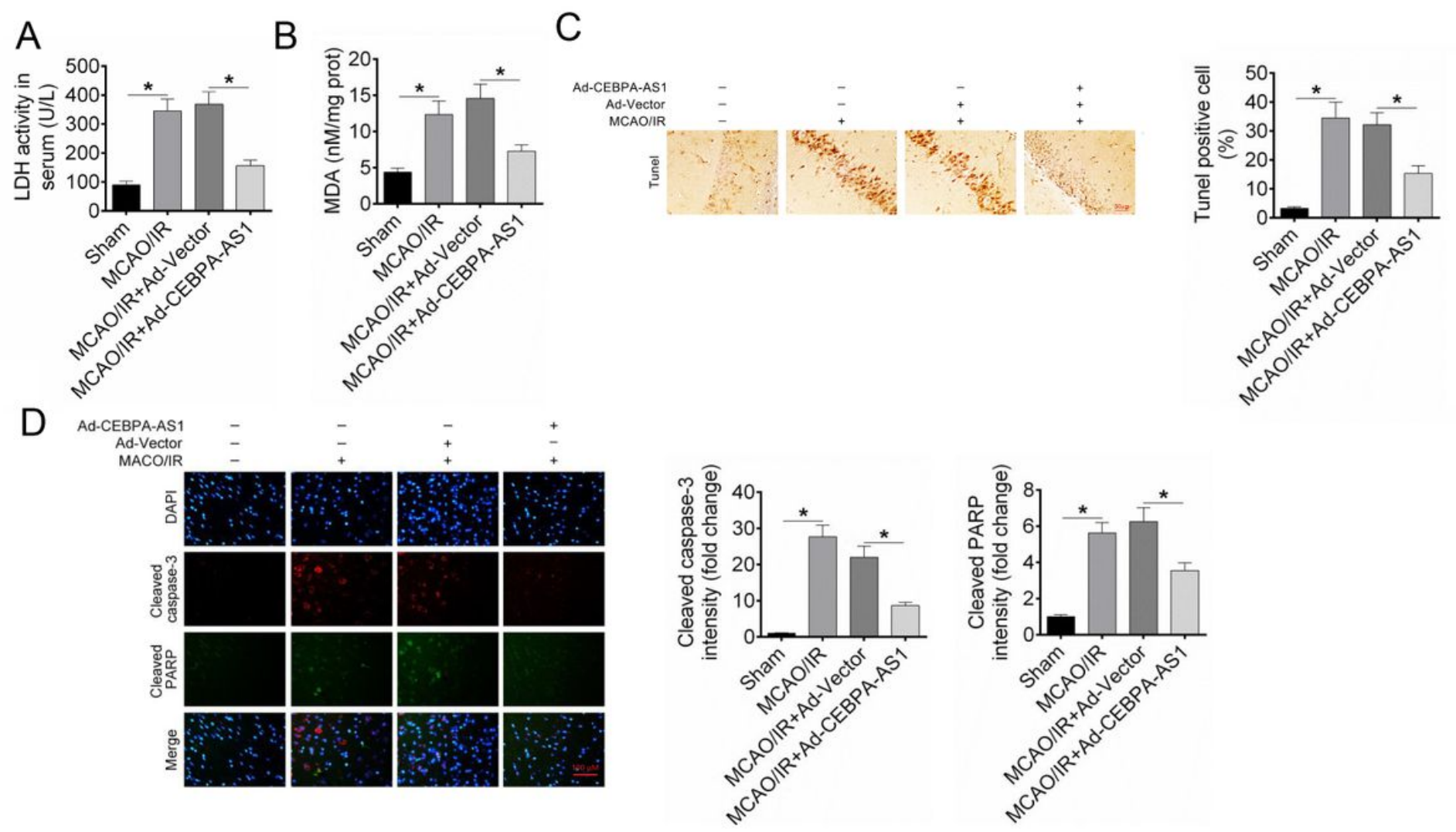

Figure 2

Increased CEBPA-AS1 expression reduced neuronal apoptosis in MCAO/IR model A. LDH kit was used to detect LDH levels; B. MDA kits were used to detect MDA levels; $C$. The apoptosis of neuronal cells were detected by TUNEL assay; D. immunofluence assay was used to detect the expression of cleavedcaspase-3 and cleaved-PARP. * $\mathrm{P}<0.05$.

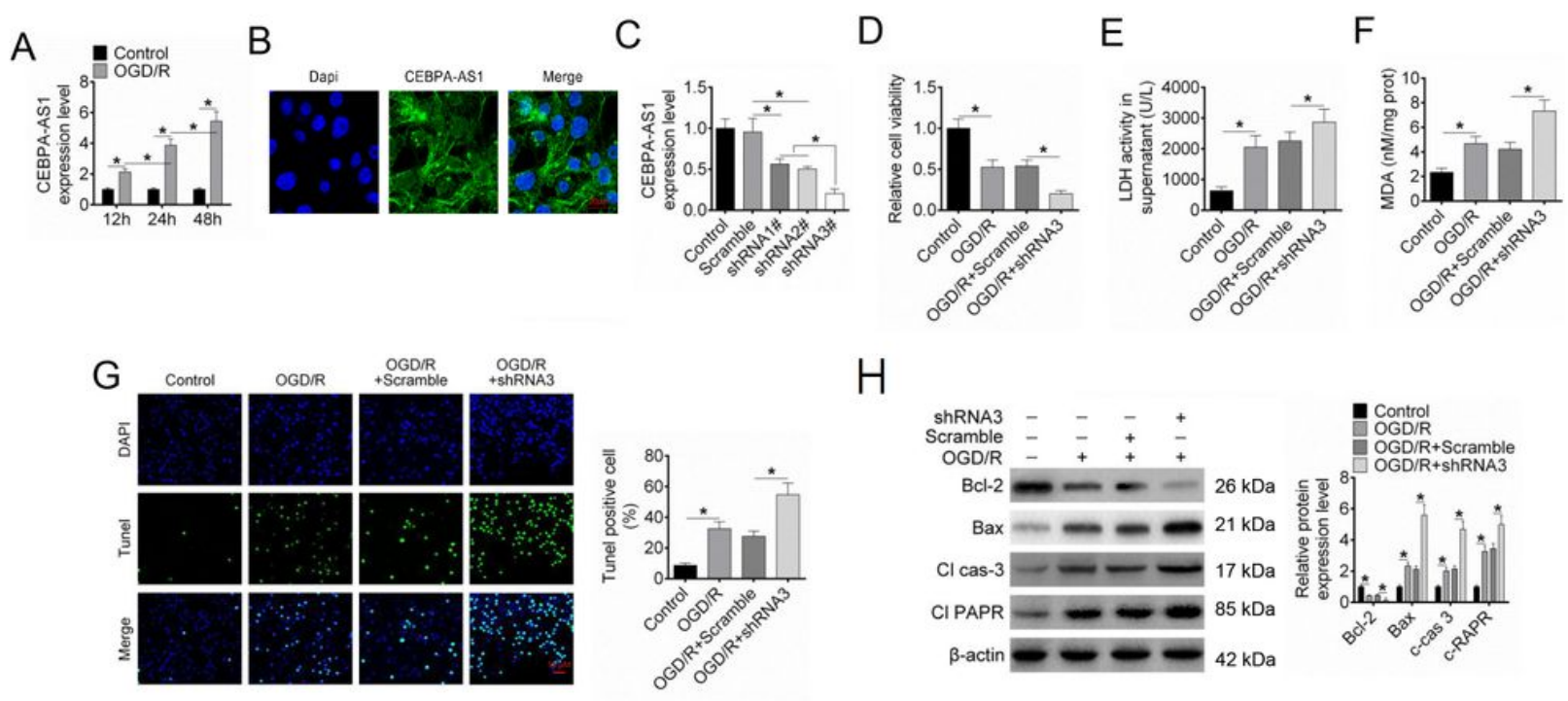

Figure 3 
Knockdown of CEBPA-AS1 aggravated cell damage OGD/R cell model A. transfection efficiency of shCEBPA-AS1; B. FISH assay for distribution of IncRNA CEBPA-AS1 in cells; C. CEBPA-AS1 expression level detection after shRNA transfection; D. Cell viability; E. LDH kit was used to detect LDH levels; F. MDA kits were used to detect MDA levels; $G$. The apoptosis of neuronal cells were detected by TUNEL assay; $H$. The protein level of Bcl-2, Bax, cleaved-caspase-3 and cleaved-PAPR. ${ }^{*} \mathrm{P}<0.05$.

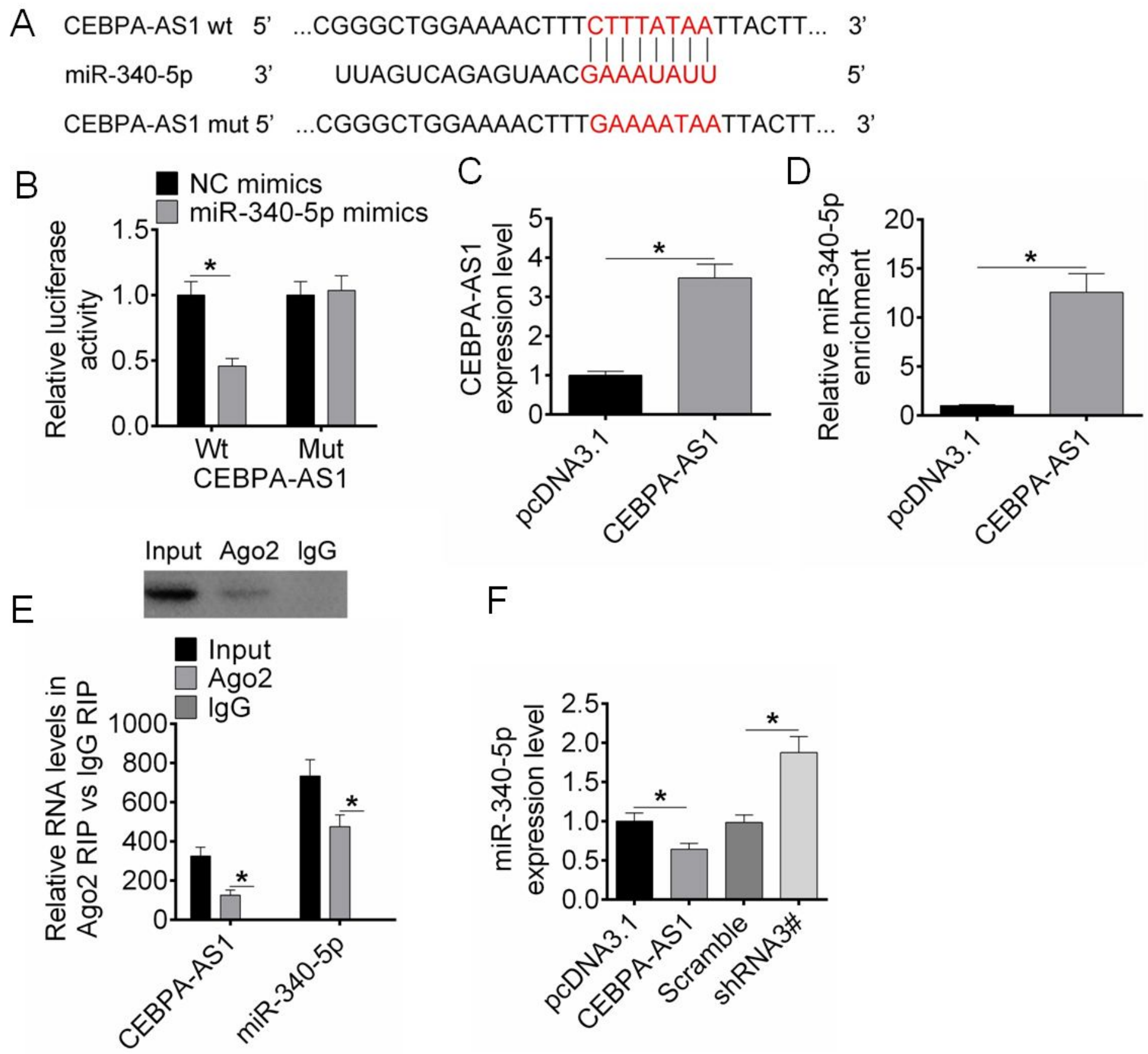

Figure 4

CEBPA-AS1 negatively regulated miR-340-5p expression as a molecular sponge A. The miR-340-5p and CEBPA-AS1 binding sites was predicted based on the miRDB website. B. Luciferase reporter system detected binding sites. C. RT-PCR assay was processed to verify the successful of CEBPA-AS1 
transfection. D. After overexpressing CEBPA-AS1 in PC-12 cells, RNA pulldown experiments were performed, and miR-340-5p enrichment was detected by qRT-PCR. E. RIP experiments were performed in PC-12 cells, and miR-340-5p, CEBPA-AS1 levels in RISC complexes were detected by qRT-PCR. F. Overexpression of CEBPA-AS1 plasmid and knockdown of shRNA were transfected into PC-12 cells, and qRT-PCR was used to detect miR-340-5p expression level. * $\mathrm{P}<0.05$.

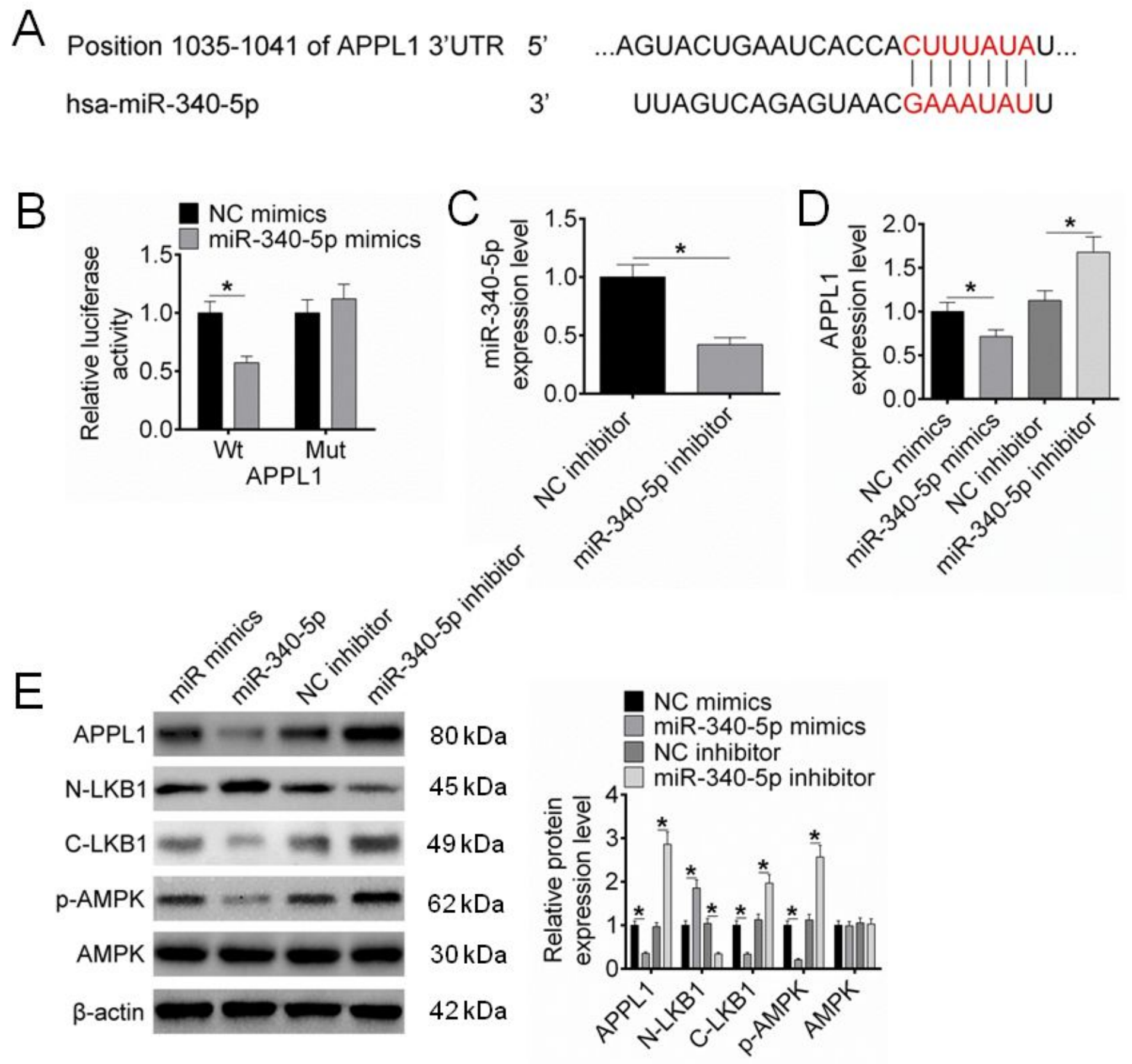

Figure 5

APPL1 was the target of miR-340-5p A. Targetscan predictED target genes binding to miR-340-5p. B. Dual luciferase reporter assay verified the binding of miR-340-5p and APPL1. C. RT-PCR assay confirmed the successful of miR-340-5p inhibitor transfection. D. The expression of APPL1 after miR-340-5p mimics or 
inhibitor transfected. E. The expression of APPL1, N-LKB1, C-LKB1, p-AMPK and AMPK after miR-340-5p mimics or inhibitor transfected. ${ }^{*} \mathrm{P}<0.05$.
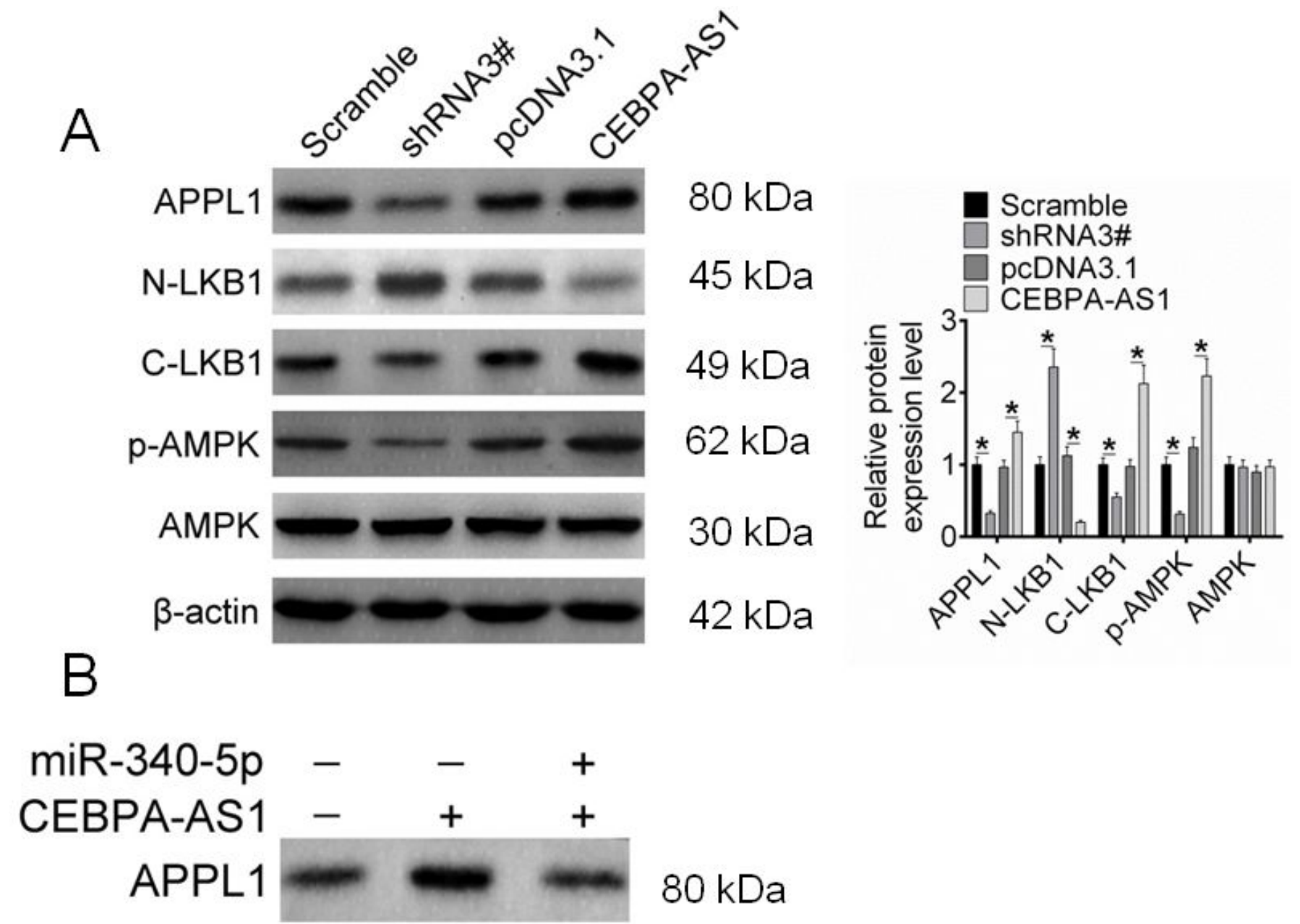

$80 \mathrm{kDa}$
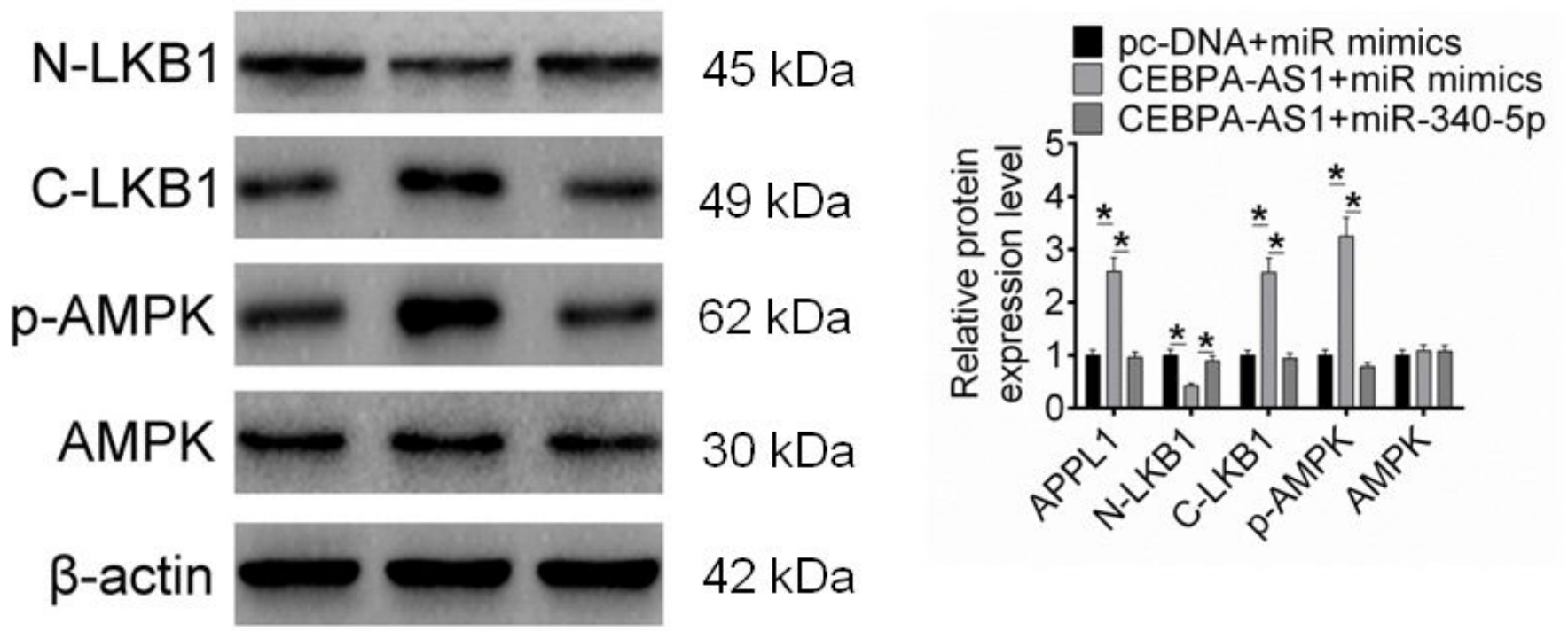

Figure 6

CEBPA-AS1 increased APPL1 expression via regulating miR-340-5p, and affected APPL1/LKB1/AMPK pathway A. After over-expression and knockdown of CEBPA-AS1 in PC-12 cells, WB detected APPL1, LKB1 (nucleus and intracellular), p-AMPK, AMPK protein levels; B. After overexpression of CEBPA-AS1 and miR- 
340-5p in PC-12 cells, WB detected APPL1, LKB1 (nucleus and intracellular), p-AMPK, AMPK protein levels. * $\mathrm{P}<0.05$.
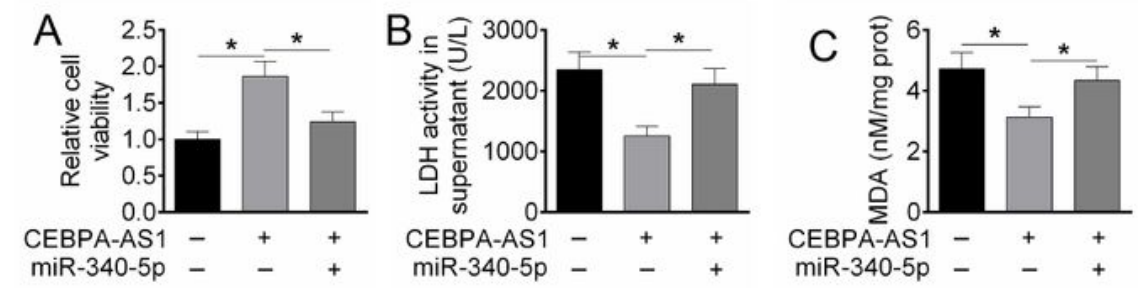

\section{D}
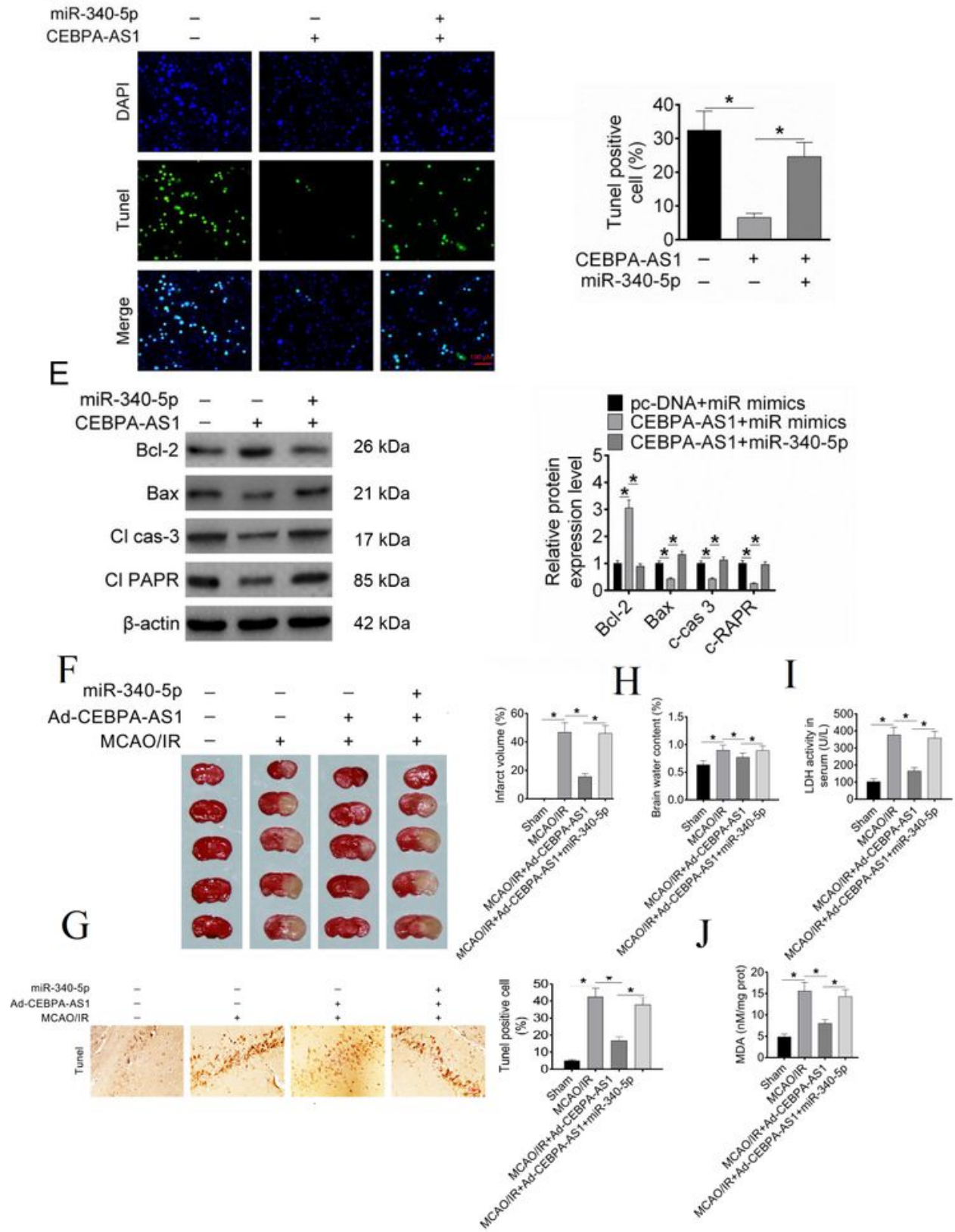

Figure 7

CEBPA-AS1 attenuated OGD/R-induced damage by reducing miR-340-5p levels A. Cell viability was detected by MTT assay; B and C. LDH and MDA content were detected by kit; D. TUNEL was used to detect apoptosis; E. WB was used to detect Bcl-2, Bax, cleaved-caspase-3 and cleaved-PAPR protein 
levels. F. TTC staining for infarct volume. G. TUNEL positive cells. H. Brain water content. I. LDH activity in serum. J. MDA level. ${ }^{\star} \mathrm{P}<0.05$. 